\title{
Report on Foundation and Organization of Econophysics Graduate Courses at Faculty of Physics of University of Warsaw and Department of Physics and Astronomy of the Wrocław University
}

\author{
R. KutneR ${ }^{a * *}$ AND D. GRECH ${ }^{b}$ \\ ${ }^{a}$ Division of Physics Education, Institute of Experimental Physics \\ Faculty of Physics, University of Warsaw \\ Smyczkowa 5/7, PL-02-678 Warsaw, Poland \\ ${ }^{b}$ Institute of Theoretical Physics, Wrocław University \\ pl. Maxa Borna 9, PL-50-204 Wrocław, Poland
}

Two different, working examples of organization of econophysics graduate courses at the Faculty of Physics, University of Warsaw and the Department of Physics and Astronomy of the Wrocław University are considered. In the first example we have a system where the interdisciplinary, econophysical education begins only after three years study of physics. Within this system the M.Sc. as well as Ph.D. theses in econophysics are conducted only at the Faculty of Physics. In the second example the B.Sc. theses in econophysics are accomplished in the Department of Physics and Astronomy again after three years study but higher degrees can be prepared either in physics in the Institute of Theoretical Physics or in economy in the Institute of Economical Sciences. M.Sc. and Ph.D. theses can also be conducted. For both examples, the graduate students of econophysics are obliged to participate in traditional (typical) economical lectures and trainings which are offered them by economical departments while lectures and trainings (tutorials and/or laboratory classes) in econophysics are offered them by physics departments themselves. Thus Poland is one of a few countries, where so modern interdisciplinary knowledge is systematically offered to students.

PACS numbers: 01.10.Hx, 89.65.Gh, 01.40.Di, 01.10.-m

\section{Essential foundation}

\subsection{Brief, subjective history of econophysics}

Since about two decades, methodologies, models and theories developed mainly within statistical physics and condensed matter physics are contributing

*corresponding author; e-mail: erka@fuw.edu.pl 
to the analysis of economical phenomena and processes. This sort of research has, since more than one decade, the official name of econophysics due to the journal Physica A as well as other physical journals (such as The European Physical Journal B or International Journal of Modern Physics C), which have open special sections under this name (or name including term complex systems) to publish some economic studies (which initially concerned mainly financial markets).

The prehistory and history of econophysics and its relation to physics and other disciplines were systematically reviewed by the physicist and economist Roehner who emphasized similarities and differences in his unique book Patterns of Speculation. A Study in Observational Econophysics [1]. Recently, a popular article Fizyk na gietdzie (Physicist on Stock Market) giving a short history of econophysics dedicated to Polish readers was also published by the physicist Hołyst [2].

Right now, the question arises of the definition of econophysics. This neologism, which was introduced in 1997 by Stanley, defined a new multidisciplinary field of activities of physicists who are working on economic problems (both in a micro- and macroscale) by applying a variety of new conceptual approaches derived from physical sciences (e.g., from statistical physics and thermodynamics, condensed matter physics, field theory or physics of chaos) [3-7]. In this way, a much deeper analysis and understanding of both economical and social phenomena and processes were obtained.

The informal beginning of econophysics can be dated by the publishing of the article entitled Lévy Walks and Enhanced Diffusion in Milan Stock Exchange by Mantegna, which appeared in Physica A (1991) [8]. In this paper Mantegna analyzed the historical series of MIB15 (Milano Italia Borsa consisting of 15 sectors) index and observed that variations of the index for different time-horizons can be described by the Lévy stable distribution instead of the Gaussian one as it might be expected from the Bachelier theory [9]. This paper inspired physicists and also communities of mathematicians to do financial modelling beyond Brownian motion (or the Wiener process), for example, to study Lévy processes [10-12], rare and extreme events on financial markets [13], and the influence of discrete scaling invariance (log-periodic oscillations) [14] as well as financial crashes [15] and fully developed turbulences [16].

A pioneer dissertation by Bachelier appeared in 1900; Bachelier was a mathematician but his advisor was Poincaré, the great mathematician and theoretical physicist. In this work Bachelier studied the dynamics of the Paris Stock Exchange index and invented a brilliant concept of mathematical formulation of a random walk on a financial market. In fact, Bachelier was the first to invent stochastic process, called later Markov stochastic process, and apply them to describe evolution (in a probabilistic sense) of the stock market index and assets. A few years later this process was applied by Smoluchowski [17] to describe and explain the physics of Brownian motion - this approach is yet canonical for the theory and 
applications of stochastic processes. Of course, the same essential physical aspects of Brownian motion were in 1905 considered by Einstein [18]. Next, developing the Bachelier concept, Samuelson discovered, in the mid of 1960s, the so-called, geometrical Brownian motion of indexes and assets on stock markets [19] and in the beginning of 1970s Black and Scholes offered the model of option dynamics, which created a new branch of mathematics called financial mathematics [20].

On the other side, at the beginning of 1960s Mandelbrot discovered fat tails in the statistics of cotton price changes quoted on commodities market [21]; this constituted the foundation for the application of fractal structures not only to commodity but also to financial markets. Later he discovered and used in this context multifractal structures [21] (and refs. therein).

The financial markets are a well-defined example of a complex system whose modelling is developed, in particular, by physicists. This development is stimulated by avalanches of easily accessible empirical data extracted from financial markets, which are continuously electronically monitored and recorded, even within the timescale of the order of miliseconds. Everything what was said above makes financial markets extremely attractive, particularly for physicists interested in a deeper understanding of the collective speculative (economic and social) phenomena.

\subsection{Polish econo- and sociophysical community}

Nowadays, both the international and Polish communities of econo- and sociophysics are already well defined. For example, in 2004 the new section Physics in Economy and Social Sciences (Fizyka w Ekonomii i Naukach Społecznych, FENS) of the Polish Physical Society (Polskie Towarzystwo Fizyczne, PTF) was founded. One can estimate that already dozens of Polish physicists from different scientific institutions, such as Faculty of Physics of Warsaw University of Technology, Faculty of Physics of University of Warsaw, Institute of Theoretical Physics of Wrocław University, Institute of Physics of Jagiellonian University, Faculty of Physics and Applied Computer Science of AGH University of Science and Technology, H. Niewodniczański Institute of Nuclear Physics of Polish Academy of Sciences, Institute of Physics of Polish Academy of Sciences, Institute of Theoretical Physics and Astrophysics of Gdańsk University, Faculty of Mathematics and Natural Sciences of Card. Stefan Wyszyński University, Institute of Physics of University of Rzeszów, Department of Econometrics and Informatics of Warsaw Agricultural University are systematically publishing econo- and/or sociophysical papers.

Since 2003 the Polish econo- and sociophysical community has organized several conferences:

(i) Europhysics Conference Applications of Physics in Financial Analysis 4, Warsaw 2003, Faculty of Physics, Warsaw University of Technology [22].

(ii) First Polish Symposium on Econo- and Sociophysics, Warsaw 2004, Faculty 
of Physics of University of Warsaw, and Faculty of Physics of Warsaw University of Technology [23].

(iii) Conference on Applications of Random Matrices to Economy and other Complex Systems, Kraków 2005, Mark Kac Complex Systems Research Center and EU Center of Excellence in Information Society Technologies "Copira" at the Institute of Physics, Jagiellonian University [24].

(iv) Second Polish Symposium on Econo- and Sociophysics, Kraków 2006, Institute of Physics, Jagiellonian University and Faculty of Physics of AGH University of Science and Technology [25].

(v) Third Polish Symposium on Econo- and Sociophysics, Wrocław 2007, Institute of Theoretical Physics, Wrocław University.

Furthermore, the International Conference on Economic Science with Heterogeneous Interacting Agents 2008 (ESHIA/WEHIA 2008), which is being organized by the Faculty of Physics of Warsaw of University of Technology, took place in June'08 in Warsaw. Previous conferences of this type took place in: Kiel (2003), Kyoto (2004), Essex (2005), Bologna (2006) and Fairfax (2007).

We believe that the reform of academic education discussed recently in Poland will be a chance for further development of the interdisciplinary branches of science, for example, such as the emerging scientific disciplines like econo- and sociophysics.

\subsection{Contribution of econophysics to economy}

So far econophysics has been contributing, roughly speaking, to four (of course, overlapping) areas of traditional and modern economy:

(1) financial markets,

(2) wealth and income distribution in various societies,

(3) industrial economics (e.g., companies' size distribution and growth rates),

(4) networks analysis,

since, an almost common belief exists among econophysicists that universal empirical regularities can be discovered at least in the above mentioned areas.

All studies both in econo- and sociophysics can be classified into three categories irrespective whether they are based on:

(i) statistical methods, including phenomenological thermodynamics of equilibrium and non-equilibrium states or the exotic (or non-Gaussian and non-exponential) statistical physics,

(ii) deterministic methods; this category is based, for example, on field theories or theory of deterministic chaos, and

(iii) hybride methods, where deterministic approaches are perturbed by different stochastic noises (Langevin type dynamics, which is supplied with a white noise, is here a reference example). 
Moreover, challenges defined by econophysics stimulated physicists to develop different theories of time-series such as the theory of statistical time-series, as well as non-linear and non-stationary ones.

The mentioned above contributions were possible thanks to that they were published mainly in the following physics and general journals: Science, Nature, Physical Review Letters, Physical Review, Physica A, The European Physical Journal, International Journal of Modern Physics C, The European Physics Communications, as well as in such financial mathematics and economics ones as Quantitative Finance, Journal of Economic Behaviour $\&$ Organization. Moreover, several prominent publishers devoted special series to these modern, interdisciplinary branches of science.

Indeed, in econophysics courses we are already able to offer students lectures including a carefully selected and systematized knowledge characteristic of all these areas and categories, including selected theories of time-series.

\section{Aims of education}

Today already several physics departments in various countries offer courses in econophysics. For example,

(a) Duke University (private university) located in Durham, North Carolina, United States,

(b) Trinity College Dublin, the University of Dublin, Ireland,

(c) University of Munster, Germany,

(d) University of Southern Denmark,

(e) University of Ulm, Sweden,

(f) University of Fribourg, Switzerland.

The essential, common feature of these courses is fact that they define econophysics as a subfield of physics, which means that the traditional definition of physics should be extended.

The aim of the courses, offered to graduate students by the Warsaw and Wrocław Universities, is to present characteristic elements of econophysics to demonstrate:

(i) a rich contribution of physicists mainly to modern economy and financial mathematics,

(ii) trends and perspectives of econophysics, and

(iii) its possible applications.

\subsection{Lectures and trainings}

Lectures and trainings (tutorials and/or laboratory classes) are split into two essentially different groups which are shown in Tables I-III. As it is seen, in Table I 
we present basic lectures and trainings offered by the Faculty of Physics of University of Warsaw, which can be classified into four different (slightly overlapping) classes: (P) physics, (E) (mainly) econophysics, (MM) mathematical methods, (C) computational methods.

TABLE I

Basic lectures and training programmes offered at the Faculty of Physics, University of Warsaw.

\begin{tabular}{c|l|c|c}
\hline \hline No. & \multicolumn{1}{|c|}{ Lecture } & Hours/week & ECTS \\
\hline P1 & Physical experiments under extreme conditions & 30 & 2.5 \\
P2 & Advanced statistical physics & 75 & 6 \\
P3 & Statistics for physicists & 60 & 5 \\
P4 & Stochastic processes in physics & 60 & 2.5 \\
P5 & Mechanics of continuous media & 75 & 6.5 \\
P6 & Electrodynamics with elements of field theory & 90 & 7.5 \\
\hline E1 & Non-Gaussian stochastic processes & 60 & \\
& $\quad$ in natural, economical and social sciences & 60 & 5 \\
E2 & Introduction to econophysics & 90 & 7.5 \\
\hline MM1 & Mathematical methods in physics & 120 & 10 \\
MM2 & Advanced time-series analysis & 60 & \\
\hline C1 & Mathematical and computer modelling & 30 & 2.5 \\
& $\quad$ in natural sciences & 30 & 2.5 \\
C2 & Computer simulations in physics by means of examples & 30 & 2.5 \\
C3 & Computational materials science & &
\end{tabular}

Moreover, graduate students must take part in a seminar on econo- and sociophysics (2 h/week, 2 ECTS).

In Table II we present the basic lectures and training programmes offered by the Faculty of Economics of University of Warsaw, within the following classes: (IE) introduction to economy, (M) microeconomics, (ME) macroeconomics, (EM) econometrics, (A) insurance, (F) financial markets, (MM) mathematical methods, while the Faculty of Mathematics, Informatics and Mechanics of University of Warsaw offers only lectures and training programmes within classes (A) and (F) presented in Table III.

As it is seen, the three faculties mentioned above offer students ten classes of lectures and trainings ${ }^{\dagger}$. Let us note that students cannot skip any of them and,

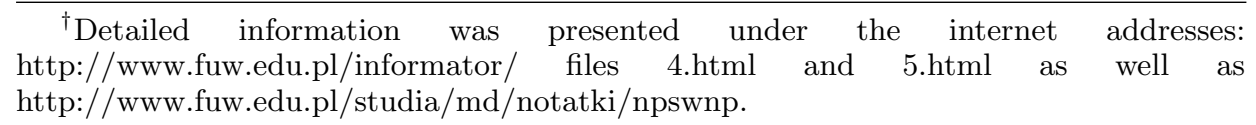


TABLE II

Basic lectures and training programmes offered at the Faculty of Economics of University of Warsaw.

\begin{tabular}{c|l|c|c}
\hline \hline No. & \multicolumn{1}{|c|}{ Lecture } & Hours/week & ECTS \\
\hline IE & Introduction to economy & 30 & 3 \\
\hline M1 & Experimental economics & 30 & 3 \\
M2 & Microeconomics & 30 & 4 \\
M3 & Advanced microeconomics & 120 & 12 \\
\hline ME1 & Macroeconomics & 60 & 7 \\
ME2 & Advanced macroeconomics & 105 & 10 \\
\hline EM & Econometrics & 120 & 9 \\
\hline A & Theory of insurance & 30 & 3 \\
\hline F & Modelling of financial markets & 30 & 4 \\
\hline MM1 & Game theory & 60 & 4 \\
MM2 & Chaos in dynamical systems & 30 & 3 \\
& & & TABLE III
\end{tabular}

Basic lectures and training programmes offered at the Faculty of Mathematics, Informatics and Mechanics of University of Warsaw.

\begin{tabular}{c|l|c|c}
\hline \hline No. & \multicolumn{1}{|c|}{ Lecture } & Hours/week & ECTS \\
\hline A & Risk theory in insurance & 60 & 6 \\
\hline F & Mathematical modelling in finance & 60 & 3.5
\end{tabular}

in principle, should choose lectures and trainings from the above given Tables. The educational trajectory of graduate students possesses, in some extent, an individual character supervised by the head of the econophysics specialization.

\subsection{Specific aspects of econophysic specializations at the Warsaw and Wroctaw Universities}

\subsubsection{Econophysics at University of Warsaw}

Today, there are two ways for graduate students to reach specialization in Methods of Physics in Economy (Econophysics) offered by the Faculty of Physics of University of Warsaw. Namely,

(1) participation in the Master Degree Programme, where after three years of study the students are obliged to choose one of the offered specializations (in this case the econophysic one), and next after two (or two and half years, which depends on the specialization regulations; in this case two years) graduate (by presenting the Master's Thesis) and obtain the M.Sc. degree, or

(2) participation in the Bachelor Degree Programme where after three years study student obtains the B.Sc. degree in physics (by presenting his Bachelor's Thesis); to continue further study toward the M.Sc. degree, the students 
have to complete (in the course of one complementary year) the courses which were missed in respect of the above mentioned Master Degree Programme.

However, this situation is temporary; soon, when the Bologne process will dominate in our educational system, these two ways will be reduced to a single one, which passes through the B.Sc. degree for all students. Hence, the concept that any specialization begins only within the Master Degree Programme (i.e., after the student conducted the Bachelor Degree Programme) will be preserved.

\subsubsection{Econophysics at Wroctaw University}

A different approach to econophysics teaching [26] is taking place at the Department of Physics and Astronomy of the Wrocław University in cooperation with the Department of Law, Administration and Economic Sciences (from the same University), where graduate students may choose econophysics specialization within the three years Bachelor Degree Programme. Students obtain the B.Sc. degree in econophysics and then may continue a two years postgraduate programme either within the Master Degree Programme in physics at the Department of Physics and Astronomy or in economy at the Department of Law, Administration and Economic Sciences. The basic concept of the Wrocław econophysics course is to give B.Sc. graduates at least two opportunities for further education. It is worth to notice that econophysics graduates in Wrocław do not have to pass any additional entry examination to become postgraduate students in economic sciences and have exactly the same rights as economy graduate students with bachelor degree in economic sciences (B.E.).

There is a plan to open in the future also master postgraduate course in econophysics for those physics graduate students who did not have any contact with econophysics so far or for our own econophysics graduates who would like to get deeper, more scientific than practical skills in application of physical methods in economy - mainly in finances.

Detailed lay-out of the econophysics course at Wrocław University has been presented elsewhere [26]. All lectures, tutorials and laboratory classes are split similarly to Warsaw programme into several main topics such as mathematics, physics, economic sciences with econophysics, computer classes (numerical methods and its practical applications) and supplementary classes. The variety of lectures proposed for students in Wrocław is similar to those in Warsaw but note that one deals in Wrocław with younger and less matured graduate students instead of postgraduate ones.

The main problem in any econophysics course usually concentrates around the choice and segregation of econophysics lecture content. The econophysics lecture with tutorials $(60+60 \mathrm{~h})$ conducted at Wrocław University has the following main ingredients:

1. Stock exchange market structure - description and properties of main stock exchange markets in the world and their indices, the construction of stock index, price weighted and capital weighted indices. 
2. Elements of classical technical and fundamental analysis of stocks.

3. Portfolio analysis - optimal and least risk portfolio and its construction.

4. Correlations between shares and portfolio taxonomy - ultrametric space, distance between shares, Minimal Spanning Tree (MST), practical analysis of data within MST.

5. Introduction to scaling in financial systems - scaling and power laws in complex systems, no scale objects, fractals, fractal dimensions, financial time series as fractals, Hurst exponent $(\mathrm{H})$, fractal nature of arithmetic and geometric Brownian motions, fractional Brownian motion (fBm), techniques used to estimate $\mathrm{H}$ exponent in time series (R/S, DFA, DMA, GPH), generation of time series with assumed scaling properties.

6. Multifractal analysis - multifractal detrended fluctuation analysis (MF-DFA), wavlet transform method (Wavelet Transform Modulus Maxima, WTMM) and their application in finances.

7. Stock market crashes - description, log-periodic oscillations and local fractal properties of financial time series.

8. Zipf law and Mandelbrot-Zipf law - application of Zipf analysis in prediction of financial events.

9. Random matrices (RM) in finance - properties of Wigner and Wischart eigenspectrum, connections with correlation matrices on stock market.

10. Statistical analysis of financial time series - Gaussian processes and central limit theorem (CLT), non-Gaussian processes and generalized central limit theorem (GCLT), stable Lévy distribution, statistics of low- and high-frequency financial data, heavy tails of probability distributions in finance, asymptotic behavior.

11. Basic concepts of physics of nonextensive systems - Boltzmann-GibbsShannon entropy vs. Tsallis entropy, Tsallis q-functions exponential and normal distributions and their applications in description of heavy tails in distribution of financial data.

12. Modelling of non-stationary time series - AR, ARMA, ARCH, GARCH processes and their application in finance.

13. Optimal investment horizon problem - Gaussian and non-Gaussian cases, comparison with real financial data.

14. Introduction to basic properties of large networks - exponential and scaleless networks, chosen applications.

15. Basic concepts of financial options and its pricing - European and American options, Ito lemma, derivation of Black-Scholes formula.

16. Microscopic (microeconomic) market models and numerical simulations spin based models. 
17. Basic concepts of game theory.

As it is seen, econophysics specializations, offered both by the Warsaw and Wrocław Universities, are organized in the way, which is complementary but the teaching programme in econophysics itself is essentially similar (although classified and organized differently).

\section{Conclusions}

We expect that graduate students in econophysics will:

(1) have ability to collaborate within interdisciplinary teams (which include, for example, economists, financial mathematicians, sociologists and social psychologists),

(2) be open for essential needs and requirements of different e.g., financial, actuary and advisory as well as scientific and educational institutions,

(3) be open to additional trainings or self-development connected with the job,

(4) have the ability to observe and deal with physical, economical and social processes and phenomena,

(5) be able to gain and elaborate (including visualization) the empirical (first of all the massive) data records,

(6) have the competence to interpret the empirical data including analytical and numerical modelling as well as computer simulation.

All these competences constitute a background for finding useful and satisfying employment.

\section{Acknowledgments}

One of us (R.K.) wishes to thank Professor Teresa Rząca-Urban for her stimulating and helpful suggestions concerning organization of econophysics graduate courses on Faculty of Physics of University of Warsaw. Also, one of us (D.G.) is grateful to Professor Marcel Ausloos from University of Liége for his several discussions concerning the program of econophysics graduate courses on Department of Physics and Astronomy of Wrocław University, as well as for his advices and support.

\section{References}

[1] B.M. Roehner, Patterns of Speculation. A Study in Observational Econophysics, Cambridge Univ. Press, Cambridge 2002.

[2] J. Hołyst, Wprost 46, 84 (2003) (in Polish).

[3] W. Paul, J. Baschnagel, Stochastic Processes. From Physics to Finance, Springer-Verlag, Berlin 1999.

[4] R.N. Mantegna, H.E. Stanley, An Introduction to Econophysics. Correlations and Complexity in Finance, Cambridge Univ. Press, Cambridge 2000. 
[5] J.-P. Bouchaud, Theory of Financial Risk. From Statistical Physics to Risk Management, Cambridge Univ. Press, Cambridge 2000.

[6] K. Ilinski, Physics of Finance. Gauge Modelling in Non-Equilibrium Pricing, Wiley Finance Series, Wiley, Chichester 2001.

[7] Econophysics and Sociophysics. Trends and Perspectives, Eds. B.K. Chakrabarti, A. Chakraborti, A. Chatterjee, Wiley-VCH Verlag, Weinheim 2006.

[8] R.N. Mantegna, Physica A 179, 232 (1991).

[9] J. Bernstein, Am. J. Phys. 73, 395 (2005).

[10] W. Schoutens, Lévy Processes in Finance. Pricing Financial Derivatives, Wiley Series in Probability and Statistics, Wiley, Chichester 2003.

[11] S.I. Boyarchenko, S.Z. Levendorskii, Non-Gaussian Merton-Black-Scholes Theory, Advanced Series on Statistical Science ESApplied Probability, World Sci., New Jersey 2002.

[12] A. Kyprianou, W. Schoutens, P. Wilmott, Exotic Option Pricing and Advanced Lévy Models, Wiley, Chichester 2005.

[13] Extreme Events in Nature and Society, Eds. S. Albeverio, V. Jentsch, H. Kantz, The Frontiers Collection, Springer-Verlag, Berlin 2006.

[14] R.N. Mantegna, H.E. Stanley, Nature 376, 46 (1995).

[15] D. Sornette, Why Stock Markets Crash. Critical Events in Complex Financial Systems, Princeton Univ. Press, Princeton 2003.

[16] J.F. Muzy, J. Delour, E. Bacry, Eur. Phys. J. B 17, 537 (2000).

[17] S. Chandrasekhar, M. Kac, R. Smoluchowski, Marian Smoluchowski. His Life and Scientific Work, Polish Scientific Publishers PWN, Warszawa 2000.

[18] A. Einstein, Ann. Phys. 17, 549 (1905).

[19] A. Weron, R. Weron, Inżynieria finansowa. Wycena instrumentów pochodnych. Symulacje komputerowe. Statystyka rynku, 2nd ed., Wydawnictwa Naukowo-Techniczne, Warszawa 1999 (in Polish).

[20] F. Black, M. Scholes, J. Polit. Econom. 81, 637 (1973).

[21] B.B. Mandelbrot, R.L. Hudson, The (mis)Behavior of Markets. A Fractal View of Risk, Ruin, and Reward, Basic Books, New York 2004.

[22] Applications of Physics in Financial Analysis 4, Eds. J.A. Hołyst, M.A. Nowak, Physica A 344 (2004).

[23] First Polish Symp. on Econo- and Sociophysics, Eds. R. Kutner, J.A. Hołyst, Acta Phys. Pol. B 36 (2005).

[24] Conf. on Applications of Random Matrices to Economy and other Complex Systems, Eds. Z. Burda, J. Jurkiewicz, M.A. Nowak, Acta Phys. Pol. B 36 (2005).

[25] 2nd Polish Symposium on Econo- and Sociophysics, Eds. Z. Burda, J.A. Hołyst, J. Jurkiewicz, K. Kułakowski, K. Malarz, M.A. Nowak, K. Życzkowski, Acta Phys. Pol. B 37 (2006).

[26] D. Grech, Physica A 344, 335 (2004). 